\title{
Documentación legal en entornos móviles
}

\author{
Legal documentation in mobile environments
}

\author{
Leandro ESCUDERO ATIENZA \\ Editorial Lex Nova (España) \\ lescudero@lexnova.es
}

\begin{abstract}
Resumen
El mundo de la movilidad es uno de los entornos actualmente más atractivos para que evolucionen las tecnologías de la información. El éxito de este ecosistema se basa en analizar las actividades más comunes que los usuarios realizan en su actividad diaria y adaptarlas al entorno móvil. Las funcionalidades se simplifican, a cambio de poder acceder a la información desde cualquier lugar, y se hace un especial esfuerzo en que la usabilidad de las aplicaciones sea buena. Aunque este tipo de tecnologías no sustituyen a los entornos tradicionales, resultan un buen complemento tanto para la vida profesional como de ocio. El presente artículo presenta cómo usar este nuevo paradigma para incluir información legal en estos entornos, a través de la explicación del proyecto que Lex Nova ha desarrollado para facilitar contenidos y servicios a los profesionales jurídicos. Se comentan las motivaciones que han llevado a abordar el proyecto, los diferentes planteamientos existentes y las opciones elegidas, las características de los productos obtenidos; y, finalmente, se repasan los resultados alcanzados hasta el momento.
\end{abstract}

Palabras clave: Movilidad. Smartphone. Tablets. Información juridica. Usabilidad.

\section{Acerca de Lex Nova}

Lex Nova es una de las empresas líderes dentro del mercado editorial jurídico español, avalada por sus más de 50 años de historia. Su principal actividad se centra en facilitar información jurídica actualizada en diversidad de formatos, dirigida, principalmente, a los profesionales del ámbito jurídico. El fondo documental con el que trabaja Lex Nova abarca las diferentes áreas del panorama normativo, siendo su principal objetivo la rapidez en el suministro de estos contenidos a sus clientes, marcando un estilo propio sustentado por el rigor y la fiabilidad.

En cifras, Lex Nova cuenta con un fondo documental de alrededor de 600.000 documentos agrupados en diferentes colecciones y áreas. Esta información es distribuida a 65.000 clientes en distintos soportes (papel, base de datos offline e internet).

\begin{abstract}
The world of mobility is currently one of the most attractive environments for the evolution of information technology. The success of this ecosystem is based on analyzing the most common activities that users perform in their daily work and adapt them to the mobile environment. The features are simplified, in exchange for access to information anywhere, and a special effort is made to achieve good usability of applications. Although these technologies do not replace traditional environments, they are a nice complement to both professionals and non professionals. This article introduces how to use this new paradigm to include legal information in these environments, through the explanation of a Lex Nova project that has been developed to provide content and services to legal professionals. The motivations that have led to the project, the different existing approaches and the choices and features of the products obtained are commented. Finally, the results achieved so far are summarised.
\end{abstract}

Keywords: Mobility. Smartphones. Tablets. Legal information. Usability.

\section{Motivación general del proyecto}

El mercado de dispositivos móviles es un mercado atractivo y en crecimiento. Según cifras de la consultora Analysys Mason, dentro de cuatro años habrá 2000 millones de "teléfonos inteligentes" (smartphones). Una vez conocidas las conclusiones del Mobile World Congress, celebrado en Barcelona a finales de febrero, ha quedado patente que tanto fabricantes de software, hardware, empresas de telecomunicaciones y los propios usuarios, muestran un gran interés por las ventajas que los entornos móviles les pueden proporcionar para su vida profesional y de ocio.

Además de los teléfonos móviles, la aparición de los tablets ha impulsado el desarrollo de este entorno. Estos dispositivos, mejoran la experiencia de usuario en lo referente a la consulta y a la lectura de documentación, navegación web y acceso a aplicaciones más específicas. 
El entorno móvil se adecúa perfectamente a la misión de Lex Nova (prestar servicios de información jurídica actualizables a profesionales) y es completamente integrable en el proceso de generación de contenidos del actual sistema editorial, que es independiente del soporte de salida.

Las principales ventajas de estos dispositivos a tener en cuenta para evaluar las necesidades y el alcance del proyecto han sido:

- La conectividad: Su capacidad para acceder a los contenidos demandados por el usuario desde cualquier lugar.

- La usabilidad (1): Son dispositivos especialmente pensados para usarlos de manera sencilla, aprovechando interfaces táctiles.

- La cotidianeidad: Estos dispositivos se han convertido en algo de uso general en nuestro día a día.

- Fácil acceso a nuevas aplicaciones: Estos entornos cuentan con el acceso a una serie de "markets" en los que se encuentran las aplicaciones disponibles para ser descargadas de manera sencilla y amigable.

\section{Objetivo y planteamiento}

El objetivo principal del proyecto es generar aplicaciones de valor en los entornos móviles, que permitan acceder a los usuarios a contenidos y servicios personalizables, para ser reconocidos como empresa de referencia en la distribución de información en sistemas móviles por los profesionales del mundo jurídico y los estudiantes universitarios.

Un aspecto estratégico en el planteamiento general del problema es el estudio del estado del arte en relación con el mundo de la movilidad. Este estudio se centra en dos partes: los sistemas operativos disponibles en el mercado y los diferentes modelos de hardware.

En cuanto a este último nos encontramos con:

- Teléfonos inteligentes (smartphones): Dispositivos táctiles con tamaños de pantalla de alrededor de 4 pulgadas y equipados con una amplia gama de aplicaciones y funcionalidades a las que hay que sumar las capacidades propias de un teléfono móvil.

- Tabletas electrónicas (tablets): Dispositivos electrónicos táctiles, con pantalla de entre 7 y 9 pulgadas, con mayores prestaciones y capacidades visuales que un teléfono inteligente o smartphone. Están equipados con un gran número de funcionalidades y aplicacio- nes, orientadas tanto al entorno profesional (ofimática, correo electrónico, agenda, contactos...) como al entretenimiento (reproductores multimedia, juegos, cámara fotográfica y de vídeo, redes sociales...).

Desde el punto de vista de los sistemas operativos el panorama es mucho más disperso:

- Android: Creado por la Open Handset Aliance (liderada por Google), con licencia software Apache de software libre y código fuente abierto. Permite a cualquier fabricante desarrollar sus productos sobre esta plataforma.

- iOS: Creado por Apple, con licencia propietaria. Su aspecto más destacable es que ha marcado tendencia en el mundo de la tecnología móvil y sigue destacando en él gracias a su interfaz de usuario y a su gran aprovechamiento de los recursos hardware del dispositivo sobre el que se ejecuta.

- Blackberry: Desarrollado por Research In Motion (RIM), con licencia propietaria. Es el sistema operativo líder en el sector empresarial, ya que está totalmente orientado al uso profesional (especialmente, el correo en movilidad).

- Windows Phone: Creado por Microsoft, con licencia propietaria. Una nueva versión del clásico sistema operativo de Windows para móviles.

Conocidos estos datos, a nivel de hardware, se entiende necesario que todo lo que se desarrolle sirva tanto para teléfonos como para tablets. Realmente, responden a necesidades diferentes y a público diferente, aunque puedan coincidir. En cuanto a los sistemas operativos, se ha tenido en cuenta el grado de penetración en el mercado, el número de aplicaciones disponibles en cada entorno y la evolución de los markets respectivos. Teniendo en cuenta estos aspectos, hemos considerado que Android e iOS son los que van a centrar la primera parte del proyecto. Por los estudios realizados, iOS tiene gran penetración en el entorno profesional, al que nos dirigimos; en cambio, Android, tiene fuerte implantación en los entonos universitarios, que también forman parte de nuestro público objetivo. En definitiva el proyecto se va a centrar en proporcionar contenidos y servicios para teléfonos y tablets con sistemas iOS y Android.

Otro de los aspectos importantes a la hora de elaborar el planteamiento estratégico del proyecto es que la producción de los contenidos y de los servicios para los entornos de movilidad no tenga un proceso diferente a la producción que ya está implantada para el resto de los so- 
portes. Por lo tanto, los contenidos estarán basados en los existentes en el fondo documental del Lex Nova, abarcando todas sus áreas y toda su tipología documental, pero organizados de una manera especial para aprovechar al máximo las posibilidades de la plataforma. Dado que en este tipo de plataformas resulta de especial importancia la manera de presentar los contenidos, un aspecto vital en el desarrollo del proyecto será el conseguir un aspecto atractivo y unas aplicaciones con alta usabilidad.

Además de los contenidos, el proyecto adaptará los servicios que actualmente se ofrecen para otras plataformas a entornos móviles. Se estudiarán las características de estos servicios actuales, se seleccionarán los más adaptables a este nuevo ecosistema y se generarán otros nuevos específicos a medida que se vayan observando necesidades.

En el proceso de adaptación y generación de contenidos es importante plantearse la tecnología que se va a utilizar para reconvertir los contenidos y los servicios para entornos móviles. En este particular nos encontramos con dos posibilidades:

- Aplicaciones Nativas (Apps): Están desarrolladas de forma específica para cada plataforma móvil:

- Rendimiento optimizado a las características de los dispositivos.

-Aspecto unificado.

- No suele ser necesario estar conectado para su uso.

- Deben desarrollarse de manera específica para cada plataforma.

- Navegación (WebApps): Enlaces a páginas web adaptadas a estos entornos:

- Es un navegador a veces enmascarado .

- Intentan emular el aspecto de las aplicaciones nativas.

-Es necesaria la conectividad de datos.

- Son universales, un solo desarrollo es válido para todas las plataformas.

El proyecto mezclará ambas posibilidades para utilizar la forma más adecuada en cada una de las ocasiones.

\section{Características de los contenidos y servicios ofrecidos}

Partiendo del planteamiento del apartado anterior, se han seleccionado contenidos y servicios que cumplen las siguientes características:

Para los contenidos se ha diseñado una aplicación con las siguientes funcionalidades:

- Contiene una colección de normas fundamentales en distintas áreas del derecho, como Derecho Administrativo, Civil, Mercantil, Laboral, Penal, Tributario...

- Las distintas normas se encuentran anotadas y concordadas con la capacidad de navegar a través de sus diferentes títulos.

- Dispone de funcionalidades de búsqueda tanto textual como a través de tesauros.

- Cuenta con utilidades como la inserción de notas, la generación de favoritos y una gestión de expedientes.

- Funciona en modo off-line.

- Se completa con un sistema de actualización de contenidos y un servicio de notificaciones.

Para los servicios se ha diseñado una suite con las siguientes características:

- Su objetivo es centralizar el acceso a los servicios que Lex Nova ha puesto disponibles para dispositivos móviles de entre su catálogo general de servicios.

- Incluye servicios tales como:

- Actualidad: diario jurídico, avances legislativos, gaceta informativa.

- Notificaciones: avisos configurables sobre ayudas y convenios colectivos.

— Utilidades: calendarios laborales, IPC, Euribor, IPREM.

-Ebook: acceso a la colección de libros electrónicos.

- La suite es configurable por parte del usuario para adaptar los servicios que contiene a sus necesidades reales.

- Se incluyen servicios tanto gratuitos como de pago.

\section{Resultado alcanzado}

El proyecto se ha puesto en marcha en varias fases, algunas de ellas todavía pendientes de conclusión, para de esta manera racionalizar la inversión de capital y recursos, pudiendo obte- 
ner retornos antes de finalizar el proyecto completo. Estas fases son las siguientes:

- Códigos de leyes fundamentales para iPhone: Esta fase ha sido completada y las aplicaciones se encuentran disponibles desde abril de 2011. El número de descargas realizadas hasta mayo de 2012 es de aproximadamente 8.000 .

- Códigos de leyes fundamentales para iPad: Se encuentran disponibles desde enero de 2012, obteniéndose un número total de descargas hasta mayo de 2012 de aproximadamente 3.000 .

- Suite de servicios para iPhone/iPad: Actualmente se encuentra en las últimas etapas de la fase de pruebas, esperando su salida al mercado para julio de 2012.

- Códigos de leyes fundamentales para sistemas Android: En junio de 2012 se ha publicado la primera aplicación para este entorno, el código de normas políticas. En este momento se están estudiando los primeros resultados e impresiones de los usuarios que ya tienen instalada la aplicación. A lo largo de ese mismo mes se pondrá a disposición del público el resto de las aplicaciones.

- Suite para sistemas Android: Esta fase se pondrá en marcha una vez finalizada su homóloga para sistemas iOS.

Para realizar un estudio de los resultados de la primera de estas fases, se ha contemplado una población de 250.000 profesionales potencialmente interesados en estos contenidos. Las estimaciones realizadas son las siguientes:

- Se estima que un $35 \%$ del total son usuarios de dispositivos smartphone.

- El $40 \%$ dispone de un modelo iPhone.

- El $70 \%$ es consumidor de aplicaciones de pago.

Según estos datos, estamos hablando de unos 25.000 usuarios interesados en este tipo de soluciones. Con los datos de descargas obtenidos, los resultados alcanzados hasta ahora muestran una penetración de un 30\% del mercado, lo que resulta un porcentaje superior a las cifras objetivo planteadas y nos refuerza en nuestra posición estratégica. Además, se ha comprobado que los usuarios no solamente se descargan las aplicaciones, sino que también, en un amplio porcentaje -alrededor del $60 \%$-, continúan actualizando los contenidos de la aplicación cada vez que se producen actualiza- ciones, aunque estas actualizaciones supongan un coste adicional.

El análisis de las opiniones de los usuarios que han utilizado las aplicaciones también nos aporta conclusiones positivas y sugerencias para incluir en nuestros procedimientos de mejora continua. Entre estas aportaciones podemos destacar:

- Se considera muy favorable que las aplicaciones contengan todas las normas relacionadas en su ámbito de aplicación, no solamente la principal. Por ejemplo, en el código de normas laborales, además del Estatuto de los Trabajadores se incluyen otras 30 normas fundamentales en ese ordenamiento.

- La navegación por los apartados de los documentos bien directamente o simplemente hojeando, permite al usuario ir directamente al contenido específico o repasar la norma simulando una experiencia habitual de lectura.

- La inmediatez en la actualización de contenidos, puesta en práctica tras las últimas modificaciones legislativas, aumentan la seguridad de los usuarios en el uso de la herramienta.

Otro dato interesante es que, desde la aparición de la versión para iPad de las aplicaciones, los usuarios se decantan más por este dispositivo que por la versión para iPhone. Evidentemente, las capacidades para la lectura y el estudio del iPad son muy superiores a las de su hermano pequeño y, aunque las posibilidades de las herramientas son equivalentes, la experiencia de uso es más agradable.

Con todo esto, la conclusión final no puede se otra que positiva. La línea de negocio demuestra tener buenas posibilidades de expansión; permite posicionar a la empresa como marca innovadora en la distribución de contenidos jurídicos; nos descubre ante un nuevo público; el cliente reconoce la utilidad del servicio; y genera los ingresos necesarios para su autofinanciación.

\section{Notas}

(1) Usabilidad: se refiere a la capacidad de un software de ser comprendido, aprendido, usado y ser atractivo para el usuario, en condiciones específicas de uso (ISO/IEC 9126).

Enviado: 2012-04-09. Versión corregida: 2012-06-12. Aceptado: 2012-07-02. 